\title{
Research on the Cloud Acceleration Method for the Compressed Sensing Algorithm under the Internet of Things
}

\author{
Wenzhun Huang ${ }^{1, a}$, Shanwen Zhang ${ }^{1, b}$ \\ ${ }^{1}$ Department of Electronic Information Engineering, Xijing University, Xi'an 710123, China \\ ahuangwenzhun@xijing.edu.cn; ${ }^{b}$ huangwenzhun@xijing.edu.cn
}

\begin{abstract}
Keywords: Internet of Things; Cloud Acceleration; Compressed Sensing; Sparse Representation; Mathematical Optimization; Cloud Computing; Hardware Deployment.
\end{abstract}

\begin{abstract}
In this paper, we conduct research on the cloud acceleration method for the compressed sensing algorithm under the environment of Internet of Things. On the Internet of things perception layer, an important part of the sensor network have been put forward a lot of security mechanisms. Now about access control for sensor networks is a closed network and IOT perception layer will have multiple sensor subnet and mobile users frequently switch between each sensor subnet. Traditional information sampling is based on the Shannon sampling theorem, it is pointed out that the sampling rate of the signal is not less than twice the highest frequency and the signal can be accurate reconstruction. The traditional sampling theorem is an important theoretical base for guidance on how to sample. Through the experimental simulation, the result proves the feasibility and effectiveness of the propose methodology. In the future, we plan to conduct more corresponding research to modify the current approach.
\end{abstract}

\section{Introduction}

The emergence of the Internet of things for smart home, intelligent medical, smart city, smart traffic, and many other applications in the field of rapid development laid a solid foundation. Because in the Internet of things with radio frequency identification devices, sensors, intelligent embedded numerous heterogeneous devices, such as running identifies, perception, processing and transmitting information of all kinds of services, so the Internet of things used by each node communication standards and the implementation of technology there is a huge difference. Under the environment of Internet of things, a lot of personal information by perception layer and environmental data collection and processing, which will contain some private data, so how to define the user's data access Internet security become a major challenge. In addition, the Internet of things of mobile users frequently lose often interact with the network times, its information security also needs to be protected, so anonymous data access become another need to achieve security objectives. On the Internet of things perception layer, an important part of the sensor network have been put forward a lot of security mechanisms. Now about access control for sensor networks is a closed network and IOT perception layer will have multiple sensor subnet and mobile users frequently switch between each sensor subnet, existing access control mechanisms are mostly unable to adapt to the complex architecture. In addition to the traditional access control mechanism is usually based on the identity or coarse-grained access control based on roles and the number of users in Internet environment and access requirements will be far more than the traditional single sensor network, need more flexible access control mechanism. Such a multidimensional polymorphism attribute data preserved evidence networking is very important to the normal operation of the industrial chain, but the existing data management method cannot effectively solve the problem of its storage and quick query [1-2].

On the differences of things under the network environment of network database with data in the process of testing, because of the huge amount of Internet database data and existing disorder, the current Internet environment, the use of the intelligent network database is not unified standard, different manufacturers have different standard of abnormal data in the database, which makes the traditional pattern recognition based database of network anomaly detection method for anomaly 
threshold Settings, unable to form a unified standard, database data quantity is huge and exists disorder, can't guarantee the detection accuracy and efficiency [3]. The Internet of things is a major change in the field of information technology, is considered to be the computer, the Internet and mobile communication network after the third wave of information industry. The Internet of things is based on the Internet extension and expansion of the network. Through the information sensing devices, in accordance with the contract agreement and connect any item with the Internet [4].

Information awareness provides a source of information for the Internet of things applications, is the foundation of the Internet of things application. Data collection is perceived to gathering data from perceptual node. The process of data collection focused on reliable transmission of data, requiring data during transmission without loss. Information awareness is the most basic form of data collection and the node will perceive data transmission through the network to the nodes. But as a result of the original perception often existing outliers in data, missing value, so at the time of data collection to the raw sensory data in data cleaning and the missing value estimation. Reliable transmission of data are the key problems of data collection, its purpose is to guarantee the data reliably transmitted to the gathering node from awareness [5-6]. At present, we mainly used in wireless sensor network multipath transmission and retransmission data redundancy transmission method to ensure the reliable transmission of the data. The purpose of information awareness is of interest to the user information, in most cases do not need to collect all the sensory data, and will gather in node will cause all data transmitted to the network load is too big, so under the condition of meet the application requirements of using data compression, data gathering and data fusion of network data processing technology which could realize efficient information awareness [7]. In the figure one, we show the illustration of compressed sensing technology.
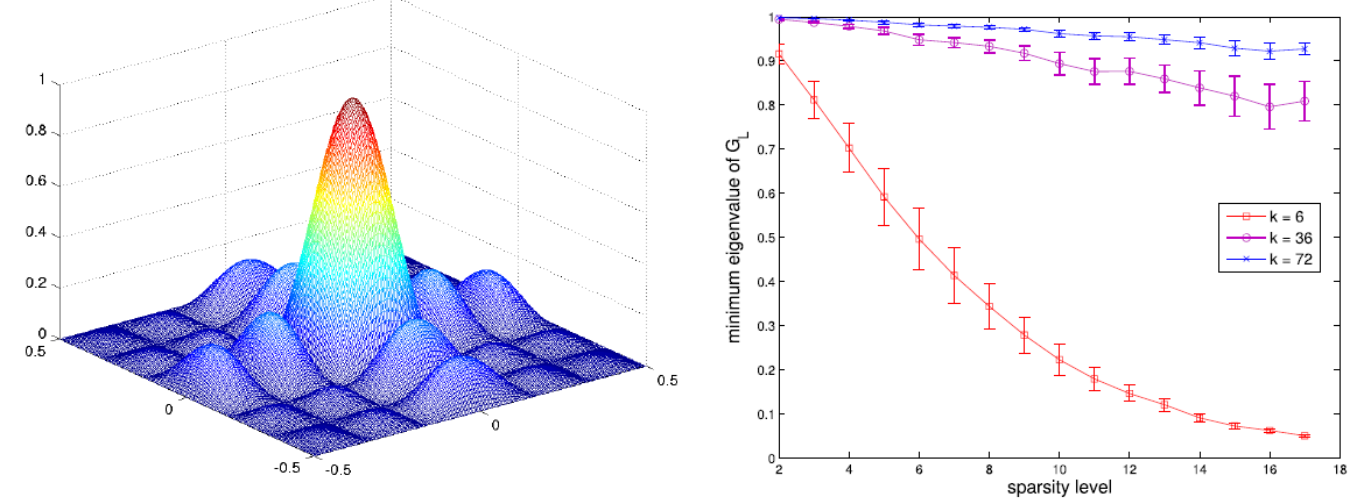

Fig. 1The Sample Illustration of Compressed Sensing Technology

To enhance the current method, we conduct research on the cloud acceleration method for the compressed sensing algorithm under the environment of Internet of Things. In order to avoid the defects of the traditional algorithm, proposed algorithm based on chaotic characteristic analysis of the differences in Internet environment network database abnormal data detection method. In Internet environment, how to detect the difference of network database with data, has become a measure differences between Internet network security is an important symbol of the current method is mainly by setting threshold, in the form of pattern recognition to complete the testing [8]. Based on related theory to build the Internet of things the difference under the environment of network database with data deviation function, provide data support for the detection of abnormal data, through the study of the minimum value of error function, abnormal data points to judge, so as to realize the differences of Internet of things environment detection of abnormal data of network database. In the following sections, we will discuss the issues in detail.

\section{The Proposed Methodology}

The Basic Concepts of Internet of Things. In order to achieve between different applications and different items between resource sharing and reuse, this paper will general, public information processing part of independence, as the general and basic Internet infrastructure in multiple Shared 
between applications. The layers could be separated into the following parts. (1) The transport layer. The transport layer is responsible for information transmission. Use technology mainly includes: all kinds of communication networks, wireless private network, the integration of Internet network, the goal is to be able to meet the demand of large, real-time sensor data transmission. (2) The smart layer. Smart layer is mainly through to the dynamic sensing information gathering, decomposition, merge and other processing and services, in the digital virtual space corresponding dynamic visual lie will be created within the physical world. On the one hand, on the Internet of things will involve in the construction of integrated management for all kinds of sensing device, to achieve reunification of the sensor technology standards, raising the share of the sensor, avoid repeated sensing information acquisition. (3) The application layer. After analysis and processing of the application layer, it will make use of the information to provide users with specific services. (4) Perception layer. Perception layer is mainly responsible for object recognition and gathering information and to implement and feedback control. Different kinds of sensor output signal, use of transport interface and the generated data format is different, in the face of the Internet of things the desired physical relationship, the goal of sharing and exchange information, how to standard output data of various sensor, allow users to identify the data is an important work. The current general solution is to packaging of the output of the sensor, produce the standardized data.

In the face of these challenges, to create a new type of information infrastructure, on the one hand, for all kinds of Internet applications and perception of equipment for all kinds of data storage, organization and management; Establish a unified, integrated application support environment on the other hand, the equipment of the interface and data standardization, and some common data processing, analysis, and other public module extracted in service way, relying on the equipment and data to quickly build applications, for items of perception, information sharing and intelligent analysis and the application of feedback control structure. From the point of the Internet of things system, at the bottom of the sensors to collect data, after transmission network transmission, to make a corresponding filter in the IOT support platform, processing, processing, analysis, formation of valuable information, to realize the feedback control, complete support for the application. From systematic perspective, on the one hand it wants to be able to collect analysis and processing the sensing information, on the other hand for the upper IOT applications provide the required sensing information. From intelligent point of view, to be able to from the vast amounts of real-time sensing, seemingly unrelated information and find out the similar relationship between data and remove redundancy, to extract the repeat mode, found that periodic variation which will provide service for prediction and decision making.

The Compressed Sensing Algorithm. With the rapid development of modern science and technology, people's demand for information is also increased. Traditional information sampling is based on the Shannon sampling theorem, it is pointed out that the sampling rate of the signal is not less than twice the highest frequency and the signal can be accurate reconstruction. The traditional sampling theorem is an important theoretical base for guidance on how to sample. It points out that the sampling rate must reach more than two times the bandwidth of the signal can be accurately reconstructed signals. With the increase of demand of information to the people, however, carry information of the signal bandwidth more and more wide, the requirement of signal processing framework based on the sampling rate and processing speed is becoming more and more high, so for the broadband signal processing difficulties in the intensifying. Currently most of the data acquisition system is based on the traditional Shannon sampling theorem to design, data taken in this way can fully represent the original signal, but they exists larger redundancy.

From the perspective of signal analysis, Fourier transform is the base of signal and the digital image processing, wavelet analysis the signal and the digital image processing into a brand-new field. After the wavelet analysis in the multi-scale geometric analysis is a new generation of signal analysis tool, it has the multi-resolution, localization and many excellent characteristics such as direction, more suitable for dealing with high-dimensional signals. These research works has laid a solid foundation for the compressed sensing theory. The theory could be formulated into the following. 


$$
\min \left\|\Psi^{T} X\right\|_{0} \quad \text { s.t. } \quad A^{C S} X=Y
$$

To another hotspot in the research of the sparse representation is signal sparse decomposition under the redundant dictionary. This is a new kind of signal representation theory: super complete redundancy function library to replace basis function, called the redundant dictionary, the dictionary elements are called atoms. In the formula two, we define the element.

$$
\|\Theta\|_{P}=\left(\sum_{i}|\Theta|^{p}\right)^{1 / p} \leq R
$$

According to the theory of compressible signals, it can be less than or is far lower than the standard way of data sampling and reconstruct the signal accurately. Unlike the Shannon theorem, compression perception is not directly measured signal itself, it uses the adaptive linear projection to obtain signal integral construction to get important information directly, ignore the information would be discarded in the loss compression. Under the theoretical framework, the sampling rate no longer depends on the bandwidth of the signal, and to a great extent, depends on two basic principles: sparse and incoherence. Compressed sensing theory is able to reverse the sampling rate, the criterion because it can fundamentally through a certain algorithm and the original signal is reconstructed from the few sample values. Reconstruction algorithm can be divided into two categories: classified into the convex relaxation method and greedy tracking algorithm.

The Cloud Acceleration Method. The definition of cloud computing has many versions, according to the definition of Wikipedia, cloud computing is a dynamic, easy to extend and being virtualized computing resources through the Internet to provide a service. The features of the cloud computing could be summarized into the following parts. (1) Distributed storage technology. The goal of distributed storage is the use of multiple servers in the cloud storage resources to meet the single server cannot meet the demand of storage. Its features are abstract representations and storage resources can be unified management. (2) Distributed computing technology. The most typical distributed computing model based on cloud platform is graphs programming model, graphs will be large tasks into many fine-grained subtasks. (3) Multi-tenancy technology. Multi-tenancy technology aims to make a large number of users can share the same stack of hardware and software resources, each user according to the need to use resources, can carry on the custom configuration of software services, and shall not affect the use of other users. (4) Elastic scale technology. Cloud computing provides a huge resource pool, and the use of the application have different load cycles, depending on the load to carry on the dynamic scaling. The application of resources will be able to significantly improve the utilization rate of resources. (5) Virtualization technology. Virtualization technology will replace the physical resource, is presented to the user with a physical resources have the same function and interface of virtual resources. May be based on an actual physical resources, can also be across multiple physical resources, the user does not need to understand the underlying physical details.

Internet of things and cloud computing can be divided into three stages: the first stage, the information and data as factors of production to use, to constantly improve the production efficiency, after combining the Internet of things and cloud computing can be obtained on the basis of the original contact more data and information, and use of cloud computing is a strong computing power and storage capacity to improve the production efficiency, to meet the various needs of the development of enterprises. In addition, after the fusion can be centralized management of all information data, through detailed analysis of data can get the conclusion and summarizes, the adjustment and optimization of the whole production system, can improve production efficiency to reduce costs. The second stage, the management and application of the information as the foundation, perfect the mode of production, with intelligent IOT technology to monitor the parts production and remote scheduling, the entire production process into intelligent, automation and centralized, effectively reduced the production error, achieve precise management. The third stage, will all kinds of information fusion, and promote the whole reconstruction and upgrading of enterprises. 
The combination of the Internet of things and cloud technology, set up a collaborative platform to assist production management, and promote the innovation of the management mode, promote the renewal and the development of technology. This pattern distribution range is relatively small, the Internet of things terminal at the center of the cloud as a data processing center, the information data are obtained terminal system has a center of the cloud for storage and processing, at the same time cloud information center will provide a unified interface to the user. Use of cloud computing is a powerful storage capacity, realizes information integration and hierarchical management, improve the efficiency of the information data management, most is applied to the community or family monitoring and as well as part of the infrastructure and so on. This model has the characteristics of large terminal, suitable for wide range of users, variety, high security requirements of the network information data. Information and application to adopt hierarchical processing model, are generally of a mass of data transmission, the requirements for information security of data transmission is lower.

Different from single center terminal model, this model is mainly used for regional span large enterprises, or for all end user information needs and Shared network can also choose to use this pattern. Application of this mode, the cloud center including public clouds and private clouds two forms, and there are no communication barriers between two forms. In under the condition of information data transmission security, realize the information sharing and transmission between the end user. In the figure three, we demonstrate the cloud combined Internet of things.

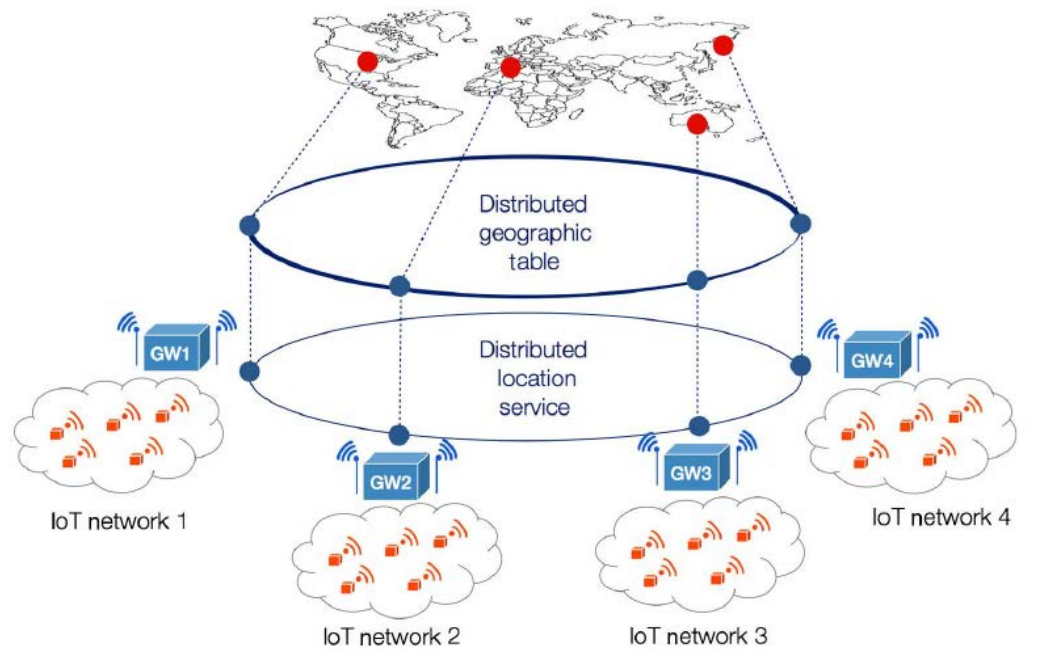

Fig. 3The Demonstration of the Cloud Combined Internet of Things

\section{Experimental Analysis and Simulation}

Internet of things technology applied in the life work is more, improve the work efficiency is of great significance. Under the background of rapid development of computer information technology, the Internet of things and the integration of cloud computing, using cloud computing with powerful computing and storage capacity, improve the Internet of things the efficiency of data transmission and computing, to promote the further development of social economy is of great significance. IOT is essentially to various sensors and a new type of existing Internet connection technology, the basis of the implementation of intelligent sensor network technology. The existence of sensor network is mainly on people's life and collection of all kinds of information, and then connected to the Internet through the network which realizes the information transmission, processing, make the content of the digital virtual has presented to the people in real life, giving people the most real perception. IOT operation platform is to support through wireless or wired network acquisition sensory information on sensor nodes. Internet compared relatively static data, under the environment of Internet of things, will be more involved in VLSI based on time and space characteristics, dynamic data calculation, and the calculation model is different in different industries. In the following figure four, we illustrate the simulation result of the proposed method compared with other state-of-the-art methods. 

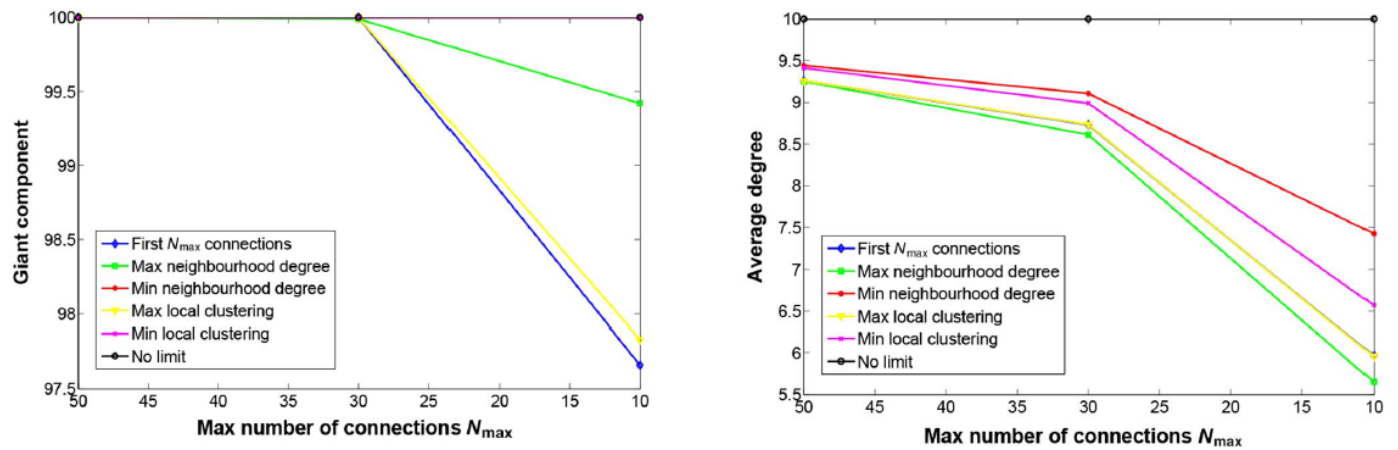

Fig. 4The Experimental Analysis and Result of the Proposed Methodology

\section{Conclusion and Summary}

In this paper, we conduct research on the cloud acceleration method for the compressed sensing algorithm under the environment of Internet of Things. Internet of each node's storage capacity and network status is different and the backup overhead, to copy storage tasks should be different. At the same time, copy the data to be accessed in one of copy distribution method on the basis of. If a user needs access to the designated area for the information, must be found within the target area and select a mobile node perception, provide the information they need for themselves, and how to choose or find the mobile node and perception interacts with become research technical difficulties. The construction of condensing subgroup is between perceived service originators and service providers to establish a trusted link, to find trust among nodes, improve the efficiency of the forwarding of a service request and finally realize the effective information transmission.

\section{References}

[1] Wang J Z, Ling C. Reliable Information Transmission Mechanism Research of the Internet of Things Sensing Layer based on the Node Authentication[J]. Netinfo Security, 2014.

[2] Wang X, Ni L. Compressed sensing data reconstruction using a modified subspace pursuit algorithm under the condition of unknown sparsity[C]// Image and Signal Processing (CISP), 2014 7th International Congress on. IEEE, 2014:1125 - 1129.

[3] He, J., Li, B., Yip, T., \& Li, J. (2014). A new secure method for the transmission of protection information between substations based on compressed sensing. PES General Meeting Conference \& Exposition, 2014 IEEE (pp.1-5). IEEE.

[4] Lin, Lanfen, Yu, Penghua, \& Li, Zeyang. (2015). Using clustering algorithm to visualize spatial-temporal internet of things data in process of agricultural product circulation. Transactions of the Chinese Society of Agricultural Engineering, volume 31, 228-235(8).

[5] Liu, B., Zhang, Z., Xu, G., Fan, H., \& Fu, Q. (2014). Energy efficient telemonitoring of physiological signals via compressed sensing: a fast algorithm and power consumption evaluation. Biomedical Signal Processing \& Control, 11(1), 80-88.

[6] Xie, F. (2015). Caca: Chinese remainder theorem based algorithm for data aggregation in internet of things on ships. Applied Mechanics \& Materials, 701-702.

[7] Suryadevara, N. K., Kelly, S., \& Mukhopadhyay, S. C. (2014). Ambient assisted living environment towards internet of things using multifarious sensors integrated with xbee platform. Smart Sensors Measurement \& Instrumentation.

[8] Zee, E. V. D., \& Scholten, H. (2014). Spatial dimensions of big data: application of geographical concepts and spatial technology to the internet of things. Studies in Computational Intelligence, 137-168. 